\title{
Effect of Thickness on the Fracture Resistance of Ceramic Partial Restorations: A Review
}

\author{
Imen Kalghoum*, Zied Feddaoui, Yosra Farhat, Nouira Zohra, Belhssan Harzallah, Mounir Cherif and Dalenda \\ Hadyaoui
}

Department of Fixed Prosthodontics, Research Laboratory of Occlusodontics and Ceramic Prostheses, University of Monastir, Monastir, Tunisia

*Corresponding author: Imen Kalghoum, Department of Fixed Prosthodontics, Research Laboratory of Occlusodontics and Ceramic Prostheses, LR16ES15, Faculty of Dental Medicine, University of Monastir, Monastir, Tunisia

\begin{abstract}
This publication describes the effect of thickness and type of material on the resistance fracture of ultra-thin ceramic restoration. The restorative phase of the treatment should not cause additional damage of the residual tooth structure. Ultrathin restorations (veneers, onlays , inlays) are considered as an alternative to traditional onlays and complete crowns. the technical aspects required for the success and the good prognosis of those new restorative design based on the control of tooth preparation with diagnostic wax-up, provisionalization, and the use of CAD-CAM technology.
\end{abstract}

Keywords: Ultrathin; restoration; ceramics; thickness; fracture

\section{Introduction}

The dental enamel is designed to withstand a lifetime. Her progressive reduction is biological consequence of advancing age. The loss of tissue may be due to the action of acidic foods, gastroesophageal reflux disease medications, and the reduction of salivary flow [1]. There are now many new protocols for a new concepts of ultrathin and non-preceramic restorations. Ceramic veneers and inlays, onlays and overlays are frequently presented as the major class of clinical conservative modalities [2]. CAD/CAM technology became popular during the last decade for the conception and fabrication of restorations. Different materials are supplied in the form of blocks that are milled to obtain the restorations [3]. Among ceramic CAD/CAM materials, lithium disilicate have recently expanded their indications to include ultra-thin restorations, with promising results. Recently, hybrid ceramic (vita Enamic) has been developed to allow faster milling of the ceramic block as ultrathin restorations with good mechanical behavior and good prognosis. Due to the importance of dental tissue preservation, it's important to evaluate the fracture resistance of reduced thickness materials made with different restorative materials $[4,5]$.

\section{Effect of Thickness}

It's very notable how well patients presenting with tooth fracture, moderate to severe loss of tooth structure when ultrathin restorations are proposed to them, both economic and biological costs are significantly lower compared to traditional and more invasive approaches. Minimal thickness can be used were in the Table $1[1,2,6-8]$. The possibility of making ultrathin $(0$, $3 \mathrm{~mm}-0,6 \mathrm{~mm}$ ) ceramic restoration allows for a more conservative preparation with minimal wear to the tooth structure. It's believed that these positive and promising results are due in part to the adhesive luting technique, dental substrate, and restorative material [13]. According to the study of Nordahi et al. comparing five thickness (0,3-0,5-0,7-1-1,5) for high-translucent(HTZ) and low -translucent (LTZ) zirconia restorations and glass ceramic (LDS) crowns. The lowest recorded load at fracture within \& mm groups was $634 \mathrm{~N}$, and $550 \mathrm{~N}$ for the Y-TZP groups at thickness of $0,5 \mathrm{~mm}$.Compared to the forces measured during mastication (approximately 5 to $364 \mathrm{~N}$ ); the results suggest the possibility to reduce restorations thickness with good prognosis especially for 
Monolithic ceramic YTZP material [9]. On the other hand, the study of JP Andrade Showed that the fracture resistance was significantly higher at a thickness of 1,5 mm compared to a thickness of 0,6 mm for veneers made of lava ultimate and vita Enamic. Manufactures of lava Ultimate, vita 3namic and IPS e.max CAD, affirmed that restorations with a minimum thickness of $1,5 \mathrm{~mm}$ on the occlusal surface of posterior teeth will support masticatory loads. Nevertheless, other studies showed that it is possible to treat severe erosive lesions or loss of wear on posterior teeth with ultrathin

Table 1.

\begin{tabular}{|c|c|c|c|}
\hline Author & Used material & Restoration (veneers/Onlay) & Minimal Thickness \\
\hline \multirow{2}{*}{ 1-Nakamura Faisuka } & *Zirconia crows (Lava plus zirconia, 3M ESPE) & -Monolithic zirconia crowns & $0.5 \mathrm{~mm}$ \\
\hline & *Lithuim discilicate crowns(IPS e max press) & -Monolithic zirconia crowns & $1.5 \mathrm{~mm}$ \\
\hline \multirow{2}{*}{ 2-Jonathon S } & ${ }^{*}$ Composite(paradigm) & -Veneers & $0.3 \mathrm{~mm}$ \\
\hline & * Hybrid ceramic(Nanoceramic) & -Veneers & $0.3 \mathrm{~mm}$ \\
\hline \multirow{2}{*}{ 3-Pascal Magne } & ${ }^{*}$ Composite resin & -Occlusal veneers & $0.6 \mathrm{~mm}$ \\
\hline & *Lithuim discilicate & -Occlusal veneers & $0.6 \mathrm{~mm}$ \\
\hline \multirow{3}{*}{ 4-JP Andrade } & *IPS e max CAD & \multirow{3}{*}{ Occlusal veneers } & $0.6 \mathrm{~mm}$ \\
\hline & *Vita Enamic & & $0.6 \mathrm{~mm}$ \\
\hline & *Lava ultimate & & $1.5 \mathrm{~mm}$ \\
\hline \multirow{2}{*}{ 5-Garqi Warg } & *Cercon zirconia & \multirow{2}{*}{-Bilayered dental ceramics } & 1,2 \\
\hline & *IPS e max Ceram & & 1,2 \\
\hline
\end{tabular}

\section{The Luting Material and Protocol}

Ceramic indirect restoration looted by the adhesive luting technique provided better fracture resistance than conventional luting technique. Hence, the use of adhesive restorations has been recommended for reinforcing the remaining dental structure because It allows intimate contact between the dental substrate, luting agent, and ceramic material, therefore occlusal forces are dissipated through the root of tooth, periodontal ligament, and alveolar bone $[3,8]$. According to many studies, associating hydrofluoric acid with silane was the most effective surface treatment with which to potentiate the bond between the ceramic and the adhesive material $[2,5,7]$. The silane enhances the chemical bond between the silicon-containing materials and the resinous material used for luting.

\section{The Type of Material $[5,7,10]$}

The study of Katrin Heck et all showed that IPS e max CAD and lava ultimate should be preferred to IPS Empress CAD for the treatment of occlusal tooth loss with ultrathin restoration, whether this result is due to the viscoelastic proprieties of the composite material [11]. In another study, Johansson et al, compared fracture resistance of monolithic zirconia and monolithic lithium disilicate after cyclic loading and thermos-cycling. they reported higher strength for zirconia restorations with the same occlusal thickness $(0,5 \mathrm{~mm}$ and $1 \mathrm{~mm})$. According to Niklas Nordhal, Ceramic materials, such as glass ceramics and zirconia show a greater scatter in (0,5-1mm) ceramic and composite resin materials [1,5]. The study of JP Andrade evaluated 0,6 mm of thickness (veneers), which are considered ultrathin restorations. On the other hand, the study of Egbert et all, shows a fracture resistance of occlusal veneers with a 0,3 mm using Paradigm MZ 100, Vita Enamic and lava Ultimate; and found promising fracture resistances. Hence, it seems that the use of use of thickness smaller than $0,6 \mathrm{~mm}$ could be used with good prognosis [3]. fracture strength compared to other material such as metal. This result calls for special factor approach when indicating reduced ceramic restoration [12].

\section{Conclusion}

Ultrathin restorations (inlay, onlays, veneers.) appear to be a promising restorative procedure in posterior and anterior teeth. The feasibility of their application depends on their fabrication options and fracture properties. Recent advances in technology and materials are offering new options for good treatment.

\section{References}

1. Feisuke Nakamura, Akio Harada, Ryoichi Inagaki, Taro Kanno, Yoshimi Niwano, et al. (2015) Fracture strength of ceramic monolithic crown systems of different thickness Acta Odontologica Scandinavica 73(8): 1-7.

2. Gaoqi Wang, BE, Song Zhang, Cuirong Bian, SMM, Hui Kong, SMM Effect of thickness ratio on load-bearing capacity of bilayered dental ceramics. Americans College of Prosthodontics 24(1): 17-24.

3. Resende TH, Reis KR, Schlichting LH, Magne P (2018) Ultrathin CADCAM ceramic occlusal veneers and anterior bilaminar veneers for the treatment of moderate dental biocorrosion: A 1,5-Year follow-up. Operative dentistry 43(4): 337-346.

4. Johnson AC, Verluis A, Tantbirojen D, Ahuja S (2014) Fracture strength of CAD/CAM composite and composite-ceramic occlusal veneers journal of prosthodontics Research 58(2): 107-114.

5. Magne P, Schilchting LH, Maia HP, Baratieri LN (2010) In vitro fatigue of CAD/CAM composite resin and ceramic posterior occlusal veneers Journal of Prosthetic Dentistry 104(3): 149-157. 
6. Jonathon S Egbert, Andrew C Johnson, Daranee Tantbirojn, Antheunis Versluis (2015) Fracture strength of ultrathin occlusal veneer restorations made from CAD/CAM composite or hybrid ceramic materials Oral Science International 12(2): 1348-8643.

7. JP Andrade, D Stona, HR Bittencourt, GA Borges, LH Burnett Junior, et al. (2018) Effect of different computer-aided design/computer-aided Manufacturing (CAD/CAM) materials and thicknesses on the fracture resistance of occlusal veneers. Operative Dentistry 43(5): 539-548.

8. Schilchting LH, Maia HP, Baratieri LN, Magne P(2011) Novel design ultra-thin CAD/CAM composite resin and ceramic occlusal veneers for the treatment of severe dental erosion. Journal of Prosthetic Dentistry 105(4): 217-226.

9. Katrin Heck, Helena Paterno, Alexander Lederer, Friederike Litzenburger Reinhard Hickel, et al. (2019) Fatigue resistance of ultrathin CAD/CAM ceramic and nanoceramic composite occlusal veneers. Science Direct $35(10)$.

10. Magne P, Stanley K, Schlichting LH (2012) Modeling of ultrathin occlusal veneers Dental Materials 28(7): 777-782.

11. Dirxen C, Blunck U, Preisser S (2013) Clinical performances of a new bomimetic double network material Open Dentistry Journal 7(6): 118122 .

12. Egbert JS, Johnson AC, Tantbirojn D, Verluis A (2015) Fracture strength of ultrathin occlusal restorations made from CAD/CAM composite or hybrid ceramic ceramic materials. Oral Science International 12(2): 5358.
To Submit Your Article Click Here:

This work is licensed under Creative Commons Attribution 4.0 License

DOI: $10.32474 /$ IPDOAJ.2020.05.000204

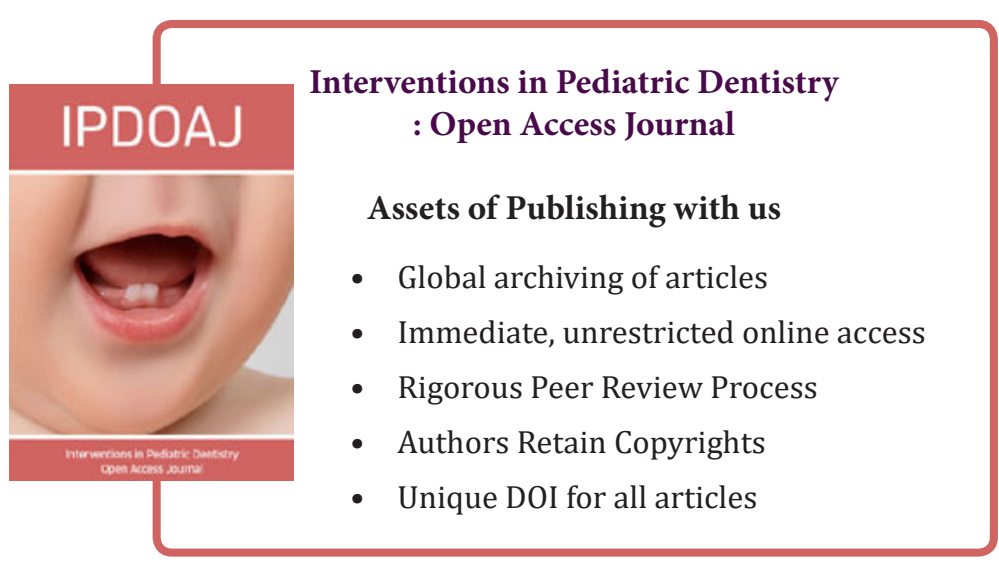

